\title{
Editorial
}

\section{Notes from the Editors of the International Journal of Developmental Science}

Welcome to the International Journal of Developmental Science (IJDS)! My co-editors and I are delighted to present the first issue of an international journal dedicated to research in developmental science, providing a forum for scientific discussion and debate. Up to volume 4 (2010), the International Journal of Developmental Science was published as the European Journal of Developmental Science (EJDS). In order to reach an international audience more effectively we decided to relaunch the Journal as International Journal of Developmental Science with the internationally established publisher AKA (internationally represented by IOS Press). The journal's aim is to present the state of the art of research in the realm of developmental science conducted in multiple disciplines worldwide. Cross-cultural as well as comparative developmental studies will also be represented.

As the field of developmental science has flourished in recent decades the abundance of research is rapidly transforming our conception of human development. The IJDS intends to provide a platform for diverse forms of studies dealing with biopsychosocial mechanisms of change, human development, and psychopathology. Without a doubt - and rightly - different conceptions and definitions of developmental science do exist. In an earlier article we defined developmental science as follows - without presuming that this definition is the "one and only":

"Developmental Science is devoted to the study of sustainable processes of the interplay of changes in individuals across the life span and of societies, considering psychological, biological, social, societal, historical and cultural factors and systems (cp. Cairns, 1996; Carolina Consortium on Human Development, 1996). (...)The interdisciplinary field of Developmental Science connects concepts and findings from various disciplines (e. g. Psychology, Anthropology, Biology, Communication Science, Neuroscience, Linguistics, Medical Science, Ethology, Philosophy and Sociology), each traditionally concerned with human and nonhuman development separately (...): Developmental Science is a metatheory rooted in developmental principles to guide work and thinking on biology and social behaviour and their interactions over ontogeny (Magnusson \& Cairns, 1996)." (Scheithauer, Niebank \& Gottlieb, 2007, p. 9)

Thus, the present journal will continue to provide an interdisciplinary and international forum for research and professional application in the field of developmental science. It aims to assemble work by researchers across different subdisciplines within psychology as well as across other disciplines such as neuroscience, linguistics, medical science, genetics etc. The reader will find original empirical or theoretical contributions, methodological papers and reviews, giving a systematic overview and evaluation of research and theories concerned with normative human development as well as developmental psychopathology during infancy, childhood, adolescence, and adulthood. All submitted and invited manuscripts pass through a multilevel peer-review process. The International Journal of Developmental Science will continue to attract internationally renowned scholars within the exciting field of 
developmental science to submit their work.

The IJDS will be published quarterly and will be available as print and online version. In addition to reviewing manuscripts submitted for publication to the journal, we will continue to compose special issues devoted to a certain topic or research question that represent the field of developmental science on a regular basis. In the past, special issues have ranged from topics such as moral development, child temperament, sibling designs and research of developmentally appropriate intervention and prevention. Internationally renowned authors in the field of developmental psychology and developmental science have already submitted manuscripts for publication and we certainly hope, they and many others will continue to do so. Finally, several distinguished researchers accepted our invitation to continue to serve on our reorganized Scientific Advisory Board, such as William M. Bukowski, Lutz Jäncke, Ulman Lindenberger, Arnold J. Sameroff, Rainer K. Silbereisen, Richard Lerner, Lars Bergman, and Dieter Wolke - just to name a few. We are particularly proud to mention that two of the most renowned founders of developmental science have served on the board of the EJDS: Paul B. Baltes ( $\dagger$ ), and Gilbert Gottlieb ( $\dagger$ ). Their contributions will continue to inspire our work for many years to come. Finally, with this first issue of the IJDS, we are very pleased to announce that Peter C. M. Molenaar accepted our invitation to join the editorial board of the journal.

We hope you will consider the IJDS as an outlet for your future research!

The Editors, Herbert Scheithauer (Editor-in-Chief), Angela Ittel, Tina Malti, and Peter C. M. Molenaar (Co-Editors).

\section{References}

Scheithauer, H., Niebank, K., \& Gottlieb, G. (2007). To see an elephant: Developmental science. European Journal of Developmental Science, 1, 6-22. 